\title{
Multidisciplinary Management of Hepatolithiasis at a Tertiary Care Center in Nepal
}

\author{
Narendra Maharjan, Paleswan Joshi Lakhey, Bikal Ghimire, Prasan BS Kansakar, Ramesh S \\ Bhandari \\ Department of GI and General Surgery, Maharajgunj Medical Campus, Tribhuvan University Teaching Hospital, \\ Institute of Medicine, Maharajgunj, Kathmandu, Nepal
}

\section{Corresponding author:}

Narendra Maharjan, MBBS, MS

Department of GI and General Surgery, Maharajgunj Medical Campus, Tribhuvan University Teaching Hospital, Institute of Medicine, Maharajgunj, Kathmandu, Nepal

Email:narendramaharjancms@gmail.com

Submitted : Jun 14, 2020

Accepted : July 29, 2020

\begin{abstract}
\section{Introduction}

Hepatolithiasis is more prevalent in Far-East than in Middle-East countries. There are various treatment modalities available for hepatolithiasis like surgical and non-surgical procedures. Non-surgical procedures have higher recurrence rate.

\section{Methods}

This is a descriptive retrospective study of the clinical data of hepatolithiasis patients treated at Tribhuvan University Teaching Hospital (TUTH), Maharajgunj from April 2016 to October 2019.

\section{Results}

There were 14 patients and mean age was $45.8 \pm 14.8$ years. It was more common in female ( $F: M=4: 3)$. Left sided hepatolithiasis was the most common type (50\%). One (7.1\%) patient had bilateral hepatolithiasis associated with unresectable cholangiocarcinoma. Eight (57.1\%) patients were treated with surgical procedures; four (28.6\%) patients underwent interventional radiological procedures and remaining two (14.3\%) patients had combined surgical and intervetional radiological procedures. Complete stone clearance was achieved in 11 patients (78.57\%). In surgical therapy group, seven out of eight (87.5\%) patients achieved the complete stone clearance; two out of four (50\%) patients in interventional radiology group and all patients in combined therapy group achieved the clearance. There was no recurrence among those patients who had complete stone clearance and there was no mortality.
\end{abstract}

\section{Conclusion}

The management of hepatolithiasis should involve multi-modality (surgical and non-surgical) therapeutic techniques available, in order to achieve complete stone clearance and prevent the complications.

Keywords: Hepatolithiasis, left lateral sectionectomy, percutaneous transhepatic biliary drainage 


\section{INTRODUCTION}

$\mathrm{H}$ epatolithiasis refers to calculi formed in biliary tree proximal to the junction of right and left hepatic ducts. ${ }^{1}$ The prevalence of hepatolithiasis is low in western countries (0.6$1.3 \%$ ) as compared to Asia-Pacific region (30$50 \%){ }^{1}$ The pathogenesis is still not well known but bile stasis, repeated biliary infection and biliary ascariasis are found to be risk factors. ${ }^{2}$ Complications like biliary stricture, liver abscess, liver cirrhosis and cholangiocarcinoma are associated with hepatolithiasis. ${ }^{3}$ There are various treatment modalities available for this disease, like hepatectomy, endoscopic and percutaneous procedures. Non-surgical procedures have got high recurrence rate upto $40 \%$ due to the residual intrahepatic biliary strictures. ${ }^{4}$ So, hepatic resection is considered as the best option as it can eliminate both the intrahepatic stones and the strictures. ${ }^{5,6}$ The main aim of all these treatments is to eradicate all stones, correct biliary strictures, remove lesions and maintain biliary flow. ${ }^{7}$ This study was conducted to demonstrate management strategies of hepatolithiasis at a tertiary center of Nepal.

\section{METHODS}

This is a retrospective study conducted in Department of Gastrointestinal and General Surgery, Tribhuvan University Teaching Hospital, Kathmandu, Nepal. All patients treated for hepatolithiasis from April 2016 to October 2019 were included. Ethical approval to conduct the study was obtained from Institutional Review Committee of Institute of Medicine, Tribhuvan University.

The patients included in this study were those requiring surgical or non-surgical treatments for the hepatolithiasis. Those patients who refused for the treatment $(n=1)$ were excluded. The diagnosis of hepatolithiasis was confirmed by contrast enhanced computed tomography (CECT) or Magnetic Resonance Chlangiopancreatography (MRCP). We recorded demography of the patients, site of hepatolithiasis, modalities of treatment and hepatolithiasis associated with cholangiocarcinoma. Outcomes measured were rate of ductal clearance, recurrence and mortality. IBM SPSS 17.0 software was used for descriptive statistical analysis and calculated mean, median, percentage and standard deviation where appropriate.

\section{RESULTS}

The total number of patients included in the study was 14 . The age of the patients ranged from 25 to 70 yrs with mean age of $45.8 \pm 14.8 \mathrm{yrs}$. It was more prevalent in middle aged people (30-50 years), i.e. $50 \%$. There was slight female predominance (F: $\mathrm{M}=$ 4:3). The mean follow-up duration was 17.9 months. Fifty percent of patients with hepatolithiasis were associated with previous biliary tract surgery. Four (36.4\%) hepatolithiasis cases were associated with previous hepaticojejunostomy, in which the most common indication was choledochal cyst (75\%) and the other being iatrogenic bile duct injury (25\%). Incidences of left, right and bilateral hepatolithiasis were $57.1 \%(n=8), 14.3 \%(n=3)$ and $28.5 \%(n=4)$ respectively. They were treated with either surgical or intervention radiological or both procedures. Eight (57.14\%) patients received surgical treatments like left lateral sectionectomy, right hepatectomy, open CBD exploration with T-tube placement and open cholecystectomy with Hepaticojejunostomy. There were four $(28.57 \%)$ patients who underwent various interventional radiological procedures like Percutaneous Transhepatic Biliary Drainage (PTBD), Balloon stricturoplasty and Dormia basket stone extraction. Two (14.2\%) patient had to undergo both the procedures to achieve complete stone clearance (Table 1).

All left lateral sectionectomy cases were done by open approach. One case of lateral sectionectomy had residual stones in right hepatic duct, identified on postoperative percutaneous transhepatic cholangiogram. The stones were cleared by pushing them with balloon catheter inserted through the PTBD tract (Fig1).

The other patient had cholelithiasis with

Table 1. Treatments received by hepatolithiasis patients

\begin{tabular}{llc}
\hline \multicolumn{1}{c}{ Treatments } & \multicolumn{1}{c}{ Procedures } & $\mathbf{N}(\%)$ \\
\hline Surgery & Left lateral sectionectomy & $3(21.4 \%)$ \\
& Right hepatectomy & $1(7.1 \%)$ \\
& Left Lateral Sectionectomy with HJ & $2(14.3 \%)$ \\
& Open CBD exploration with T-tube placement & $1(7.1 \%)$ \\
& Open cholecystectomy with HJ & $1(7.1 \%)$ \\
Intervention radiological & Percutaneous transhepatic biliary drainage & $1(7.1 \%)$ \\
procedure & Balloon stricturoplasty & $2(14.3 \%)$ \\
& Dormia basket stone extraction & $1(7.1 \%)$ \\
Combined procedure & Left Lateral sectionectomy with Balloon pushing of stone & $1(7.1 \%)$ \\
& Open CBD exploration with T-tube cholangiogram and stone extraction & $1(7.1 \%)$ \\
\hline
\end{tabular}




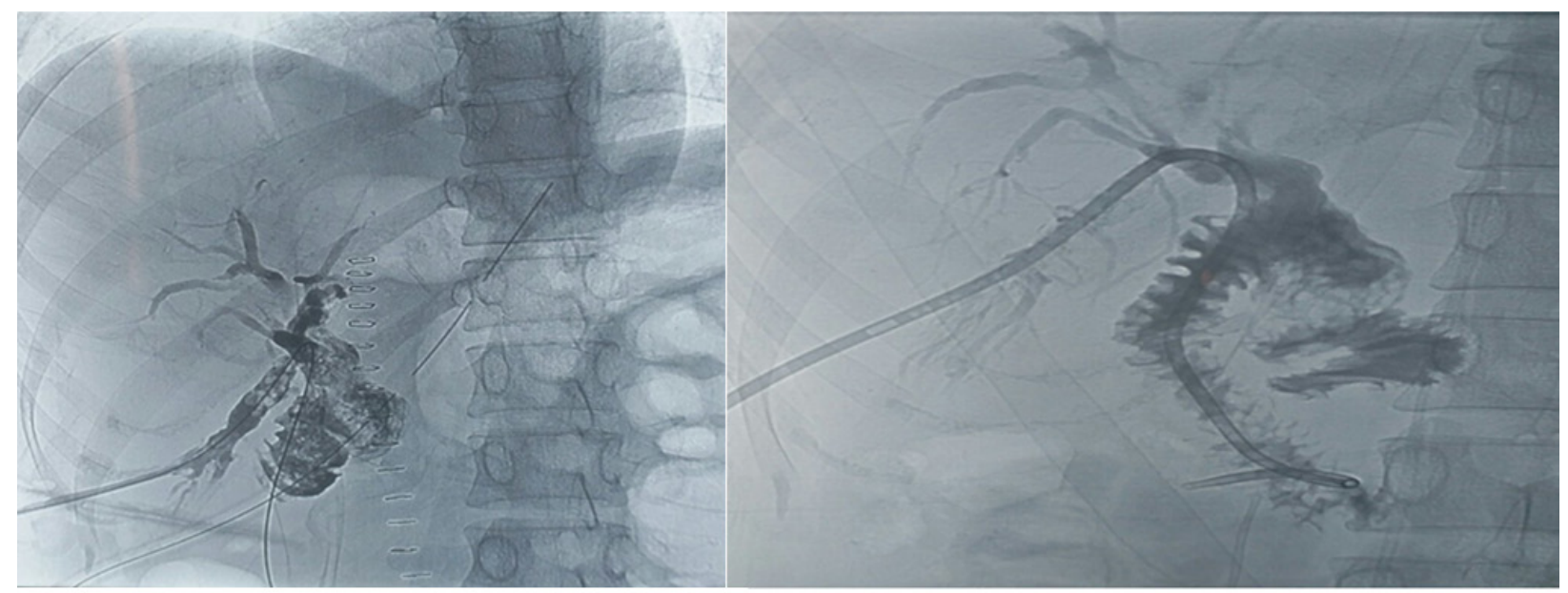

a

b

Fig 1a. Percutaneous Transhepatic Cholangiogram showing residual stones in right posterior hepatic duct after left lateral sectionectomy. Fig $1 \mathrm{~b}$. Complete stone clearance achieved in the same patient after pushing the stones using balloon catheter by Interventional Radiologist post-operatively

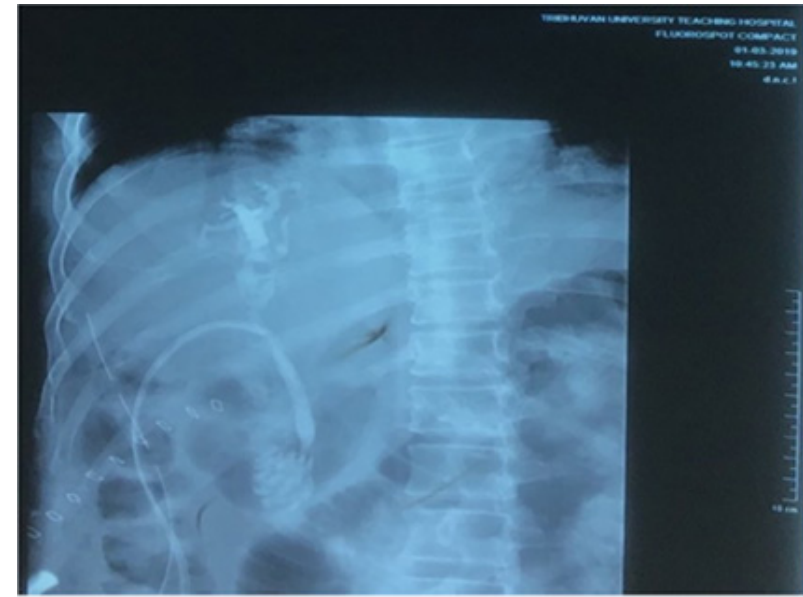

a

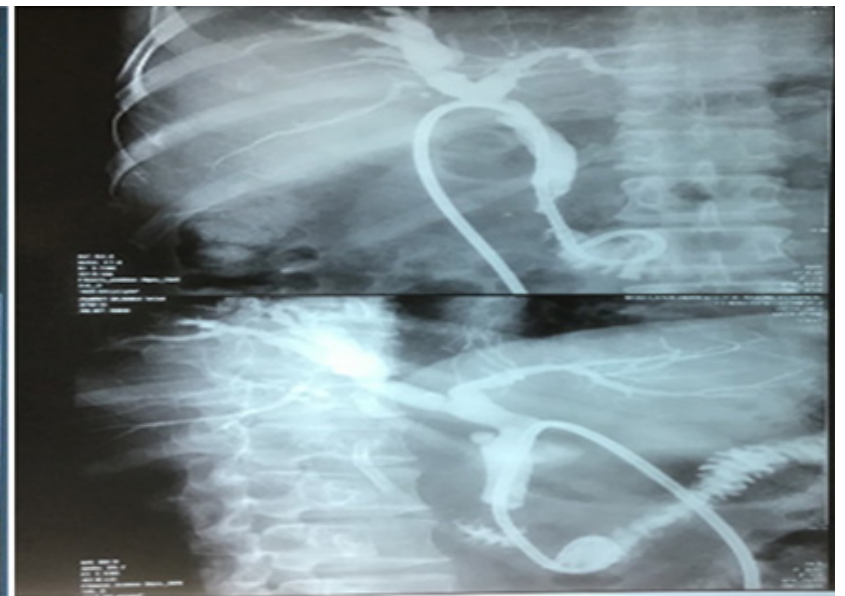

$\mathrm{b}$

Fig 2a. T-tube Cholangiogram showing filling defect in right hepatic duct. Fig 2b. Complete stone clearance achieved in the same patient after stone extraction via T-tube tract

choledocholithiasis and right hepatolithiasis. He underwent open cholecystectomy with CBD exploration. The stones in right hepatic duct were extracted via the choledochotomy site using desjardan's forcep and T-tube was placed. On postoperative period, T-tube cholangiogram showed residual stones in right hepatic duct. After 12 weeks of operation, intervention radiologists extracted the residual stones via T-tube tract and final cholangiogram showed complete stone clearance (Fig 2).

Two patients underwent balloon stricturoplasty via PTBD tract for stricture at hepaticojejunostomy site. The indications for undergoing hepaticojejunostomy were choledochal cyst and bile duct injury. Intervention radiologist pushed the stones in hepatic ducts through the hepaticojejunostomy site into jejunum. Thus complete stone clearance was achieved in both the patients.
One patient $(7.14 \%)$ had bilateral hepatolithiasis associated with unresectable intrahepatic cholangiocarcinoma, received palliative treatment with PTBD.

In overall, complete stone clearance was achieved in 11 patients (78.57\%). Seven out of 8 patients (87.5\%) who underwent surgical treatment had complete stone clearance. All patients who underwent combined procedures also had complete stone clearance. However, only 2 out of 4 patients (50\%) receiving interventional radiological procedures had the clearance. There was no recurrence among those patients who had complete stone clearance. Three patients $(21.43 \%)$ did not achieve complete stone clearance. Among them, one was associated with cholangiocarcinoma and was receiving palliative treatment with PTBD; another case had been planned for left lateral sectionectomy and third one planned for stone extraction via T-tube tract. There was no mortality. 


\section{DISCUSSION}

In this study, hepatolithiasis was found to occur in different age groups, but predominantly occurred in middle age groups. It is similar to that observed by Pandit $\mathrm{N}$ et al in their study. ${ }^{8}$ This early age at presentation of hepatolithiasis may be because of low socioeconomic conditions, malnutrition and recurrent subclinical infections of the biliary tree. ${ }^{1}$

Hepatolithiasis was found to occur more frequently in left liver $(50 \%)$ in our study. It may be because left hepatic duct joins common hepatic duct at an acute angle leading to bile stasis when associated with strictures. ${ }^{1}$ Left liver was found to be the most common site of hepatolithiasis with incidences ranging from 35.35 to $56.6 \%$. 9,10

We found that half of the patients had hepatolithiasis due to previous bile duct surgery, most commonly for choledochal cyst. This showed that bile duct surgery and choledochal cyst are risk factors for developing hepatolithiasis and such cases should be followed up properly to detect hepatolithiasis as early as possible. Kim JH et al reported that $24.24 \%$ of patients, who underwent bile duct excision with bilioenteric anastosomosis for choledochal cyst, developed hepatolithiasis in postoperative period. ${ }^{11}$

In Asian countries, the reported incidence of cholangiocarcinoma in hepatolithiasis patients ranged from 2.1 to $16 \% .{ }^{12-14}$ We had $7.4 \%$ of patients having hepatolithiasis associated cholangiocarcinoma. While in western countries, the incidence was reported to be $14.3 \% .{ }^{15}$ Thus though the incidence of hepatolithiasis in western countries was low, the incidence of hepatolithiasis associated cholangiocarcinoma was similar in the west and the east. The pathogenesis of cholangiocarcinoma secondary to hepatolithiasis is not yet well understood. However, it was postulated that chronic or recurrent cholangitis in hepatolithiasis patients caused prolong inflammation of bile duct epithelium, leading to cholangiocarcinoma. ${ }^{16}$ Thus hepatolithiasis cases must be evaluated carefully to detect the presence of cholangiocarcinoma.

The treatment of hepatolithiasis can be broadly divided into surgical therapy (Hepatic resection, CBD exploration and T-tube placement) and non- surgical therapy (extraction of stones under radiological or endoscopic guidance). ${ }^{1}$ Among them, hepatic resection is the best treatment options as it eliminates both the stones and strictured bile ducts, thereby preventing recurrence. However hepatic resection is feasible only when the disease is localized, i.e. in cases of Dong's type I (Localized stone disease: unilobar or bilobar) and type Ilb (Diffuse stone disease with segmental atrophy and/or stricture). ${ }^{17}$ In the present study, six $(42.85 \%)$ patients having localized disease underwent hepatic resections. They had complete stone clearance and did not have any recurrent stones in follow up. The recurrence of stones after treatment of hepatolithiasis is one of the troublesome complications. With median follow up duration of 8 years, the stone recurrence rate had been reported up to $30.9 \%$ after non-surgical and surgical treatments. ${ }^{18}$ In this present study, there were no recurrences after achieving complete stone clearance, with mean follow up duration of 17.9 months.

\section{CONCLUSION}

The management of hepatolithiasis should involve multi-modality (surgical and non-surgical) therapeutic techniques available, in order to achieve complete stone clearance and prevent the complications.

\section{CONFLICT OF INTEREST}

None declared.

\section{REFERENCES}

1. Sakpal SV, Babel N, Chamberlain RS. Surgical management of hepatolithiasis. HPB : the official journal of the International Hepato Pancreato Biliary Association. 2009 May; 11(3):194-202.

2. Williams EJ, Green J, Beckingham I, Parks R, Martin D, Lombard M, et al. Guidelines on the management of common bile duct stones (CBDS). Gut. 2008 Jul;57(7):1004-21.

3. Uenishi T, Hamba H, Takemura S, Oba K, Ogawa M, Yamamoto T, et al. Outcomes of hepatic resection for hepatolithiasis. American journal of surgery. 2009 Aug;198(2):199-202.

4. Ma WJ, Zhou Y, Yang Q, Li FY, Shrestha A, Mao H. The puzzle and challenge in treating hepatolithiasis. Surgical laparoscopy, endoscopy \& percutaneous techniques. 2015 Feb;25(1):94-5.

5. Li H, Zheng J, Cai JY, Li SH, Zhang JB, Wang XM, et al. Laparoscopic VS open hepatectomy for hepatolithiasis: An updated systematic review and meta-analysis. World journal of gastroenterology. 2017 Nov 21;23(43):7791-806.

6. Lei J, Huang J, Yang X, Zhang Y, Yao K. Minimally invasive surgery versus open hepatectomy for hepatolithiasis: A systematic review and meta analysis. International journal of surgery. 2018 Mar; $51: 191-8$

7. Huang ZQ, Xu LN, Yang T, Zhang WZ, Huang XQ, Cai SW, et al. Hepatic resection: an analysis of the impact of operative and perioperative factors on morbidity and mortality rates in 2008 consecutive hepatectomy cases. Chinese medical journal. 2009 Oct 5;122(19):2268-77.

8. Pandit N, Awale L, Yadav TN, Deo KB, Adhikary S. Treatment outcome of hepatolithiasis: Nepalese experience. Int Surg J. 2020;7:994-7

9. Suzuki Y, Mori T, Yokoyama M, Kim S, Momose H, Matsuki R, et al. A proposed severity classification system for hepatolithiasis based on an analysis of prognostic factors in a Japanese patient cohort. Journal of gastroenterology. $2018 \mathrm{Jul} ; 53(7): 854-60$.

10. Das BC, Rahman MM, Biswas AK, Shahriar S, Ahmed A, Khan ZR. Surgical Treatment of Hepatolithiasis: Our Experience in Bangabandhu Sheikh Mujib Medical University. Mymensingh medical journal : MMJ. 2017 Oct;26(4):797-804

11. Kim JH, Choi TY, Han JH, Yoo BM, Kim JH, Hong J, et al. Risk factors of postoperative anastomotic stricture after excision of choledochal cysts with hepaticojejunostomy. Journal of gastrointestinal surgery : official journal of the Society for Surgery of the Alimentary Tract. 
2008 May; 12(5):822-8.

12. Herman $P$, Bacchella T, Pugliese $V$, Montagnini AL, Machado MA, da Cunha JE, et al. Non-Oriental primary intrahepatic lithiasis: experience with 48 cases. World journal of surgery. 2005 Jul;29(7):858-62; discussion 63-4.

13. Chen DW, Tung-Ping Poon R, Liu CL, Fan ST, Wong J. Immediate and long-term outcomes of hepatectomy for hepatolithiasis. Surgery. 2004 Apr;135(4):386-93.

14. Liu CL, Fan ST, Wong J. Primary biliary stones: diagnosis and management. World journal of surgery. 1998 Nov;22(11):1162-6.

15. Guglielmi A, Ruzzenente A, Valdegamberi A, Bagante F, Conci S, Pinna AD, et al. Hepatolithiasis-associated cholangiocarcinoma: results from a multi-institutional national database on a case series of 23 patients. European journal of surgical oncology : the journal of the European Society of Surgical Oncology and the British Association of Surgical Oncology. 2014 May;40(5):567-75.

16. Kim HJ, Kim JS, Joo MK, Lee BJ, Kim JH, Yeon JE, et al. Hepatolithiasis and intrahepatic cholangiocarcinoma: A review. World journal of gastroenterology. 2015 Dec 28;21(48):13418-31.

17. Feng X, Zheng S, Xia F, Ma K, Wang S, Bie P, et al. Classification and management of hepatolithiasis: A high-volume, singlecenter's experience. Intractable \& rare diseases research. 2012 Nov; 1(4):151-6.

18. Cheon YK, Cho YD, Moon JH, Lee JS, Shim CS. Evaluation of longterm results and recurrent factors after operative and nonoperative treatment for hepatolithiasis. Surgery. 2009 Nov; 146(5):843-53. 